\title{
Fms-related tyrosine kinase 3 ligand promotes proliferation of placenta amnion and chorion mesenchymal stem cells in vitro
}

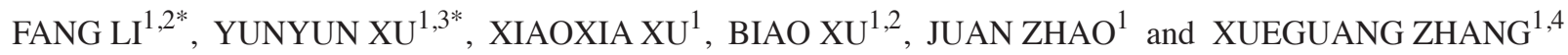 \\ ${ }^{1}$ Institute of Medical Biotechnology, Soochow University, Jiangsu Key Laboratory of Stem Cells, Suzhou, Jiangsu 215007; \\ ${ }^{2}$ Department of Human Anatomy, Histology and Embryology, School of Biology and Basic Medical Sciences, \\ Soochow University, Suzhou, Jiangsu 215123; ${ }^{3}$ Institute of Pediatrics, Children's Hospital Affliated to Soochow University, \\ Suzhou, Jiangsu 215003; ${ }^{4}$ Jiangsu Institute of Clinical Immunology, The First Affiliated Hospital of Soochow University, \\ Suzhou, Jiangsu 215007, P.R. China
}

Received July 3, 2013; Accepted March 26, 2013

DOI: $10.3892 / \mathrm{mmr} .2014 .2220$

\begin{abstract}
Placental mesenchymal stem cells (PMSCs) have important biological properties and the potential for application in numerous clinical fields, including hematopoietic stem cell transplantation and myocardial repair. There are two types of MSCs in the placenta, amniotic mesenchymal stem cells (AMSCs) and chorion mesenchymal stem cells (CMSCs). By comparing the biological characteristics of human placental AMSCs with CMSCs, the present study identified that CD90- and CD166-positive cells were located in the amniotic stroma and chorion stroma surrounding the vessels. In addition, the cultured AMSCs and CMSCs expressed high levels of CD73, CD90, CD105, CD29 and CD44; however they did not express CD14, CD34, CD45 and HLA-DR. Furthermore, the amplification of the fms-related tyrosine kinase 3 ligand (FL) in AMSCs and CMSCs was investigated in vitro. The results demonstrated that FL is able to promote the proliferation of AMSCs and CMSCs effectively in vitro, particularly that of CMSCs. In the FL group, the phenotype and the ability of AMSCs and CMSCs to differentiate into mesenchymal lineages did not change. Flt3, the receptor of FL, is expressed in AMSCs and CMSCs. In conclusion, mesenchymal stem cells with low immunogenicity were identified in the placental amniotic membrane and around the chorion axis. Furthermore, FL has a positive effect on the proliferation of AMSCs and CMSCs in vitro; however, does not affect their differentiation potential. It is
\end{abstract}

Correspondence to: Professor Xueguang Zhang, Jiangsu Institute of Clinical Immunology, The First Affiliated Hospital of Soochow University, 708 Renmin Road, Suzhou, Jiangsu 215007, P.R. China E-mail: xueguangzh@163.com

\section{${ }^{*}$ Contributed equally}

Key words: fms-related tyrosine kinase 3 ligand, amniotic mesenchymal stem cells, chorion mesenchymal stem cells, proliferation particularly promising that FL is able to stimulate CMSCs to proliferate in vitro.

\section{Introduction}

Mesenchymal stem cells (MSCs) are stem cells with multiple differentiation ability, which have great potential for clinical applications. Current research mainly focuses on human bone marrow mesenchymal stem cells (1). Human bone marrow mesenchymal stem cells are usually obtained through bone marrow puncture; however, the quantity markedly decreases along with increasing age, which limits investigations and applications of human bone marrow mesenchymal stem cells (2). Therefore, current research explores alternative sources of mesenchymal stem cells, including the peripheral blood (3), umbilical cord blood (4,5), baby teeth (6), umbilical cord (7) and placenta (8). The placenta consists of amnion, chorion and matrix decidua, and among them, the chorion is composed of nourishing cells and tissue that originates from extra-embryonic mesoderm, gathering large amounts of mesenchymal stem cells. As the placenta is a waste product following pregnancy, it has a wide source, is easy to obtain and there is no ethical debate associated with its use. Therefore, the placenta has become the focus of stem cell studies in the last decade (6-11). At an international human placenta mesenchymal stem cell research conference in 2007 , the use of human placental mesenchymal stem cells was approved for clinical application (10). The placenta has become the focus of mesenchymal stem cell research; however, the stem cell content in these tissues is limited. Therefore, optimizing the existing mesenchymal stem cell culture conditions and expanding the limited mesenchymal stem cells rapidly in vitro is crucial.

Fms-related tyrosine kinase 3 ligand (FL) is a type of cell factor which is able to promote the generation and differentiation of hematopoietic stem cells and their progenitor cells (12). Previous studies have demonstrated that the mRNA of FL was highly expressed in certain tissues, including peripheral blood mononuclear cells, the heart, placenta, lung, spleen and thymus tissue, and is also expressed to a certain extent in non-hematopoietic tissues, including the prostate, testis, ovaries, intestines, liver, kidney and skeletal muscle. Following combination with 
its receptor, Flt3, FL delivers a mitosis promotion signal to the cells to regulate cell growth and differentiation.

Therefore, on the basis of stable cultivation of human AMSCs and CMSCs, the present study investigated the importance of cell factors (FL) on the proliferation of AMSCs and CMSCs in vitro.

\section{Materials and methods}

Antibodies and reagents. The materials used included: A placenta from cesarean delivery (agreed to by the puerpera), low-glucose Dulbecco's modified Eagle's medium (L-DMEM; HyClone, Loughborough, UK), fetal calf serum (HyClone), type IV collagen enzyme (Sigma, St. Louis, MO, USA), pancreatic enzyme (Sigma), EDTA (Sigma), recombinant human FL (R\&D systems, Minneapolis, MN, USA), recombinant human alkaline fibroblast growth factor (bFGF; R\&D systems), mouse anti-human monoclonal antibodies for flow cytometry, including CD90 (BD Biosciences, Franklin Lakes, NJ, USA), CD105 (Biolegend, San Diego, CA, USA), CD73 (BD Biosciences), CD14 (Biolegend), CD34 (BD Biosciences), CD45 (Biolegend) and HLA-DR (eBioscience, San Diego, CA, USA), mouse anti-human monoclonal antibodies for immunofluorescence staining, including CD90 (eBiosience) and CD166 (eBiosience), an avidin biotin complex (ABC) kit (Boster Biological Tech Ltd., Fremont, CA, USA), perilipin (PLIN), aggrecan (ACAN), runt related transcription factor 2 (RUNX2), Flt3 and $\beta$-actin primer sequences (Genscript, Nanjing, Jiangsu, China), an Olympus BX51 light microscope (Olympus, Tokyo, Japan), a $\mathrm{CO}_{2}$ incubator (Jouan, Winchester, VA, USA) and an FC500 flow cytometer (Beckman Coulter, Miami, FL, USA). The present study was approved by the ethics committee of Soochow University (Suzhou, Jiangsu, China) and written informed consent was obtained from the patient.

Immunofluorescence stain of placental tissue. After the frozen placental tissue sections were slightly air dried, a drop of the appropriate primary antibodies (CD90; concentration of 1:400; CD166 concentration of 1:200 and phosphate-buffered saline (PBS) as the negative control) was added. Next, they were placed into a $37^{\circ} \mathrm{C}$ water bath box for $1 \mathrm{~h}$ and washed three times with PBS (3 min each time) in order to remove unbound antibodies. Sheep anti-rat fluorescence antibody combined with fluorescein isothiocyanate (FITC; at the concentration of 1:100) was added and then placed into a $37^{\circ} \mathrm{C}$ water bath box for $30 \mathrm{~min}$, washed with PBS three times ( 3 min each time) and observed under an Olympus BH-2 fluorescent microscope (Olympus).

Isolation and culture of human AMSCs and CMSCs. The cesarean section placenta and the amniotic tissue were rinsed repeatedly in PBS and the placental amniotic membranes were cut into small pieces. They were then digested in $0.25 \%$ pancreatic enzyme at $37^{\circ} \mathrm{C}$ for $10 \mathrm{~min}$ and filtered through a 100 mesh screen. The tyrosine-digested tissues were fully cleaned in PBS, continued to digest in $0.1 \%$ collagen IV enzyme for $15 \mathrm{~min}$ at $37^{\circ} \mathrm{C}$ and suspended in L-DMEM containing $10 \%$ fetal calf serum by pipetting up and down. The suspension was then filtered through a 100 mesh screen to remove any adherent cells, centrifuged at $400 \mathrm{~g}$ for $10 \mathrm{~min}$ and then the supernatant was discarded. Next, the cells sourced from the amniotic membrane were collected and divided into three groups to be subjected to different cultural conditions. Group I was cultured in complete L-DMEM containing 10\% fetal calf serum, group II was cultured in complete L-DMEM containing $5 \mathrm{ng} / \mathrm{ml} \mathrm{bFGF}$ and group III was cultured with complete L-DMEM complete containing $75 \mathrm{ng} / \mathrm{ml}$ FL.

CMSCs separation and culture in vitro. The cesarean section placenta and the cut chorion tissue were rinsed repeatedly with PBS. Once the blood was washed away, the chorion tissues were cut into small pieces and digested for $30 \mathrm{~min}$ at $37^{\circ} \mathrm{C}$ with $0.1 \%$ type IV collagenase according to the manufacturer's instructions, and CMSCs were obtained. The two types of MSCs (AMSCs and CMSCs) were cultured at $37^{\circ} \mathrm{C}$ with $5 \% \mathrm{CO}_{2}$. The medium was changed every 3 days. Following 12-14 days, the cells were passaged using conventional methods.

Flow cytometric analysis of the cellular phenotype. The AMSCs and CMSCs of the experimental groups I, II and III, which were cultured for more than three passages, were collected, respectively and the cell density was adjusted to $5 \times 10^{5}$ cells $/ 50 \mu \mathrm{l}$ with PBS containing $1 \%$ fetal calf serum. Then, rat anti-human monoclonal antibodies CD73-PE, CD90-PE, CD105-PE, CD29-PE, CD44-PE, CD14-PE, CD34-FITC, CD45-PE and HLA-DR-PE were added, respectively, and the cells were incubated at $4^{\circ} \mathrm{C}$ for $20 \mathrm{~min}$. The cells were then washed twice with PBS and finally, the cell phenotype was detected by flow cytometry.

Detection of the role of cell factors in the proliferation of AMSCs and CMSCs by cell counting. Passage three AMSCs and CMSCs from the experimental groups I, II and III were collected and seeded in 24-well plates at a density of $1 \times 10^{4}$ cells per well, respectively. The cells were treated with $75 \mathrm{ng} / \mathrm{ml}$ human FL, $5 \mathrm{ng} / \mathrm{ml}$ human bFGF or no factor for the control group. On the second day, three wells were digested for cell counting. Cells were counted using the Merck Millipore Scepter 2.0 cell counter (Merck Millipore, Darmstadt, Germany). An eight-day cell growth curve was generated with, the cell count as the y-coordinate and the culture time as the $\mathrm{x}$-coordinate.

Analysis of the differentiation potential of AMSCs and CMSCs by specific staining. The passage three AMSCs and CMSCs from the FL group were collected and seeded in a six-well plate. After the cells were attached completely, the culture medium was removed by aspiration. Fat cell inducing medium (containing $10 \% \mathrm{FBS}, 1 \mu \mathrm{mol} / \mathrm{ml}$ of dexamethasone, $0.5 \mathrm{mmol} / \mathrm{l}$ of 3 -isobutyl-1-methyl xanthine, $10 \mu \mathrm{g} / \mathrm{ml}$ of insulin and $0.2 \mathrm{mmol} / 1$ of indomethacin DMEM), bone cell inducing medium (100 nM of dexamethasone, $10 \mathrm{nM}$ of $\beta$-glycerophosphate and $0.25 \mathrm{nM}$ of phospho-L-ascorbic acid trisodium salt) and cartilage cell inducing medium $(0.5 \mu \mathrm{g} / \mathrm{ml}$ of insulin, $50 \mu \mathrm{M}$ of ascorbic acid-2 phosphate and $2 \mu \mathrm{g} / \mathrm{ml}$ of TGF- $\beta$ ) was added. Negative control cells were conventionally cultured at $37^{\circ} \mathrm{C}$ with $5 \% \mathrm{CO}_{2}$. The culture solution was changed every three days. Following 2-3 weeks for the corre- 
sponding detection, cells were observed under an Olympus BX51 light microscope (Olympus) and images were captured.

qPCR analysis of the mRNA levels of PLIN (fat cells), ACAN (cartilage cells) and RUNX2 (bone cells) in induced AMSCs and CMSCs. The third passage AMSCs and CMSCs of FL groups were collected and fat, cartilage, bone cell inducing medium and $1 \mathrm{ml}$ of TRIzol (Thermo Fisher Scientific, Carlsbad, CA, USA) was added, respectively, according to the manufacturer's instructions. The total RNA was extracted and then the random primers were transcribed from RNA into cDNA. The following primers were used in the present study: PLIN forward, 5'-AAACAGCATCAGCGTTCCCATC-3' and reverse, 5'-AGTGTTGGCAGCAAATTCCG-3', fragment length, 173 bp; RUNX2 forward, 5'-CGCAAAACCACAGAACCACAAGTGCG-3', and reverse, 5'-GTTGGTCTCGGTGGCTGGTAG-3', fragment length, $164 \mathrm{bp}$; ACAN forward, 5'-CGGGTCTCACTGCCCAACTACCCG-3' and reverse, 5'-GCCTTTCACCACGACTTCCAG-3', fragment length, $200 \mathrm{bp} ; \beta$-actin forward, 5'-ATCCTCACCCTGAAGTACC-3' and reverse, 5'-CTCCTTAATGTCACGCACG-3', fragment length, $480 \mathrm{bp}$. The PCR cycle parameters were as follows: $94^{\circ} \mathrm{C}$ for $30 \mathrm{sec}, 56^{\circ} \mathrm{C}$ for $30 \mathrm{sec}$ and $68^{\circ} \mathrm{C}$ for $60 \mathrm{sec}(32$ cycles). $\beta$-actin expression was used as a control for expression quantity analysis. The PCR cycle parameters for $\beta$-actin were as follows: $94^{\circ} \mathrm{C}$ for $3 \mathrm{~min}, 94^{\circ} \mathrm{C}$ for $30 \mathrm{sec}, 58^{\circ} \mathrm{C}$ for $30 \mathrm{sec}$ and $72^{\circ} \mathrm{C}$ for $45 \mathrm{sec}$ ( 30 cycles). The PCR products were detected by agarose gel electrophoresis.

Analysis of the Flt3 mRNA levels in human AMSCs and CMSCs. Passage three AMSCs and CMSCs of three different samples were collected and $1 \mathrm{ml}$ TRIzol was added, respectively, according to the manufacturer's instructions. Then the total RNA was extracted and the random primers were transcribed from RNA into cDNA. The PCR cycle parameters were as follows: $95^{\circ} \mathrm{C}$ for $3 \mathrm{~min}, 95^{\circ} \mathrm{C}$ for $30 \mathrm{sec}, 65^{\circ} \mathrm{C}$ for $30 \mathrm{sec}, 72^{\circ} \mathrm{C}$ for $60 \mathrm{sec}(35 \mathrm{cycles})$ and $72^{\circ} \mathrm{C}$ for $10 \mathrm{~min}$. The Flt 3 primer sequence was used in the present study as follows: Flt3 forward, 5'-TCAAGTGCTGTGCATACAATTCCC-3' and reverse, 5'-CACCTGTACCATCTGTAGCTGGCT-3', fragment length, $176 \mathrm{bp}$. The PCR products were detected by agarose gel electrophoresis.

Statistical analysis. All the data were analyzed with SPSS 10.0 (SPSS, Inc., Chicago, IL, USA). P $<0.05$ was considered to indicate a statistically significant difference. All values are expressed as the mean \pm standard deviation. Fisher's t-test was performed among all groups.

\section{Results}

Distribution of MSCs in the amniotic and chorionic membranes of human placenta. In order to identify the distribution of MSCs in human placenta, the expression of CD90 and CD166 was separately examined by immunofluorescence analysis. The immunofluorescence stain demonstrated that CD90- and CD166-positive cells of amniotic membrane tissue were distributed mainly in the amniotic epithelial and amniotic membrane stroma, while the positive cells of

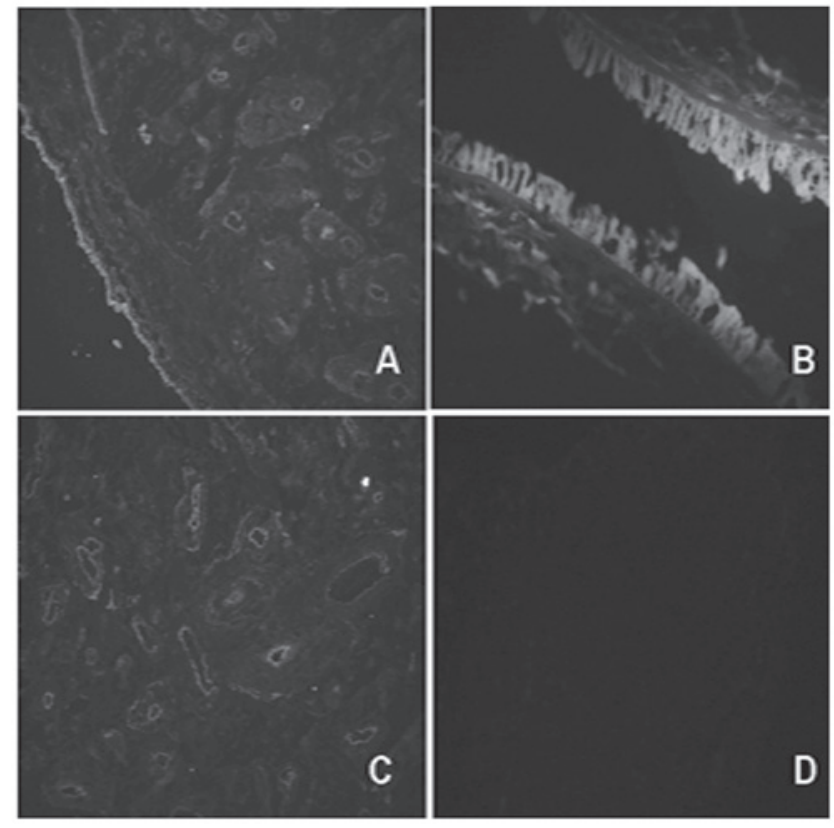

Figure 1. Immunofluorescence stain of the distribution of CD90 and CD166 positive cells in the placenta. (A) CD90 positive cells distributed mainly in the amniotic epithelial and amniotic membrane stroma (magnification, $x 40$ ); (B) CD166 positive cells distributed mainly in the amniotic epithelial and amniotic membrane stroma (magnification, x100); (C) CD166 positive cells were mainly in the vascular endothelium and stroma near the blood vessels, (magnification, x40); (D) Negative control.

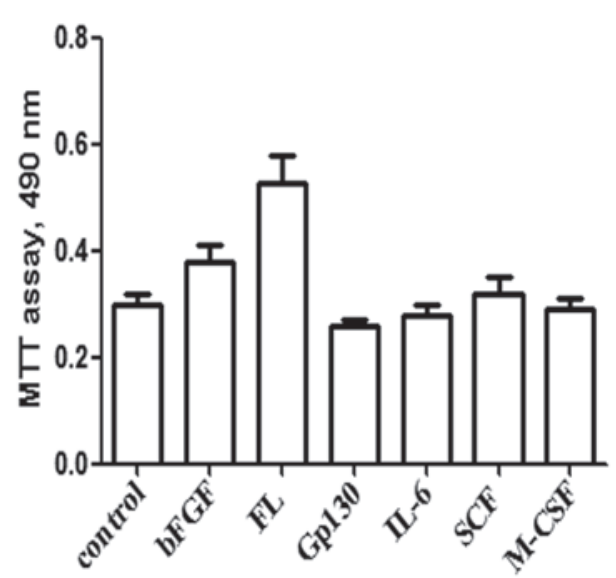

Figure 2. Different cytokines affect the proliferation of placental mesenchymal stem cells. FL, fms-related tyrosine kinase 3 ligand; bFGF, fibroblast growth factor; IL-6, interleukin-6; SCF, stem cell factor; M-CSF, macrophage colony stimulating factor.

the chorionic membrane tissue were mainly in the vascular endothelium and stroma near the blood vessels (Fig. 1). This indicated that human placenta amniotic and chorionic membranes are tissues which are rich in MSCs.

Biological characteristics of AMSCs and CMSCs from human placenta. In order to optimize the protocol for growing MSCs, the following cytokines were used: bFGF, FL, Gp130, interleukin (IL)-6, stem cell factor (SCF) and macrophage colony stimulating factor (M-CSF) (Fig. 2). AMSCs and CMSCs were mainly cultured from human placenta by enzymatic digestion and using an adherent 
Table I. Flow cytometric analysis of AMSCs.

\begin{tabular}{|c|c|c|c|c|c|c|c|}
\hline $\begin{array}{l}\text { Maker } \\
\text { group }\end{array}$ & CD73 & CD90 & CD105 & CD14 & CD34 & CD45 & HLA-DR \\
\hline Control & $62.6 \pm 2.52$ & $84 \pm 1.00$ & $78 \pm 1.00$ & $0.2 \pm 0.10$ & $0.13 \pm 0.05$ & $0.17 \pm 0.06$ & $0.27 \pm 0.05$ \\
\hline $5 \mathrm{ng} / \mathrm{ml} \mathrm{bFGF}$ & $71.3 \pm 1.53$ & $93.3 \pm 1.52$ & $81.4 \pm 0.53$ & $0.13 \pm 0.06$ & $0.23 \pm 0.07$ & $0.23 \pm 0.05$ & $0.5 \pm 0.10$ \\
\hline 75 ng/ml FL & $69.7 \pm 1.54$ & $95 \pm 1.00$ & $86 \pm 1.00$ & $0.23 \pm 0.07$ & $0.33 \pm 0.06$ & $0.27 \pm 0.05$ & $0.23 \pm 0.08$ \\
\hline
\end{tabular}

AMSCs, AMSCs, amniotic mesenchymal stem cells; FL, fms-related tyrosine kinase 3 ligand; bFGF, fibroblast growth factor.

Table II. Flow cytometric analysis of CMSCs.

\begin{tabular}{|c|c|c|c|c|c|c|c|}
\hline $\begin{array}{l}\text { Maker } \\
\text { group }\end{array}$ & CD73 & CD90 & CD105 & CD14 & CD34 & CD45 & HLA-DR \\
\hline Control & $70 \pm 1.00$ & $85 \pm 1.00$ & $81.7 \pm 1.53$ & $0.27 \pm 0.06$ & $0.23 \pm 0.15$ & $0.33 \pm 0.16$ & $0.47 \pm 0.15$ \\
\hline $5 \mathrm{ng} / \mathrm{ml}$ bFGF & $82.3 \pm 0.58$ & $97.3 \pm 1.53$ & $95 \pm 1.00$ & $0.63 \pm 0.15$ & $0.47 \pm 0.25$ & $0.5 \pm 0.36$ & $0.47 \pm 0.21$ \\
\hline 75 ng/ml FL & $79.7 \pm 1.53$ & $97 \pm 1.00$ & $91 \pm 1.00$ & $0.43 \pm 0.21$ & $0.6 \pm 0.20$ & $0.5 \pm 0.20$ & $0.4 \pm 0.20$ \\
\hline
\end{tabular}

CMSCs, chorion mesenchymal stem cells; FL, fms-related tyrosine kinase 3 ligand; bFGF, fibroblast growth factor.
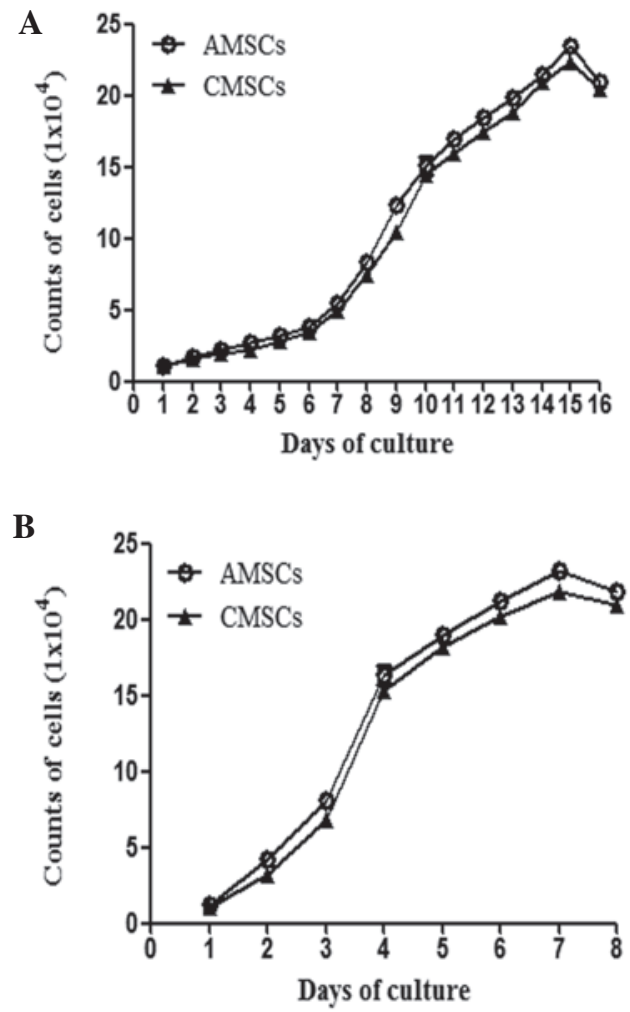

Figure 3. Growth curves of AMSCs and CMSCs cultured in vitro. (A) Growth curves of primary AMSCs and CMSCs; (B) growth curves of passage AMSCs and CMSCs. AMSCs, amniotic mesenchymal stem cells; CMSCs, chorion mesenchymal stem cells.

culture method, and then the primary generations of AMSCs and CMSCs were selected to obtain values for the 16-day growth curve. It was confirmed that AMSCs and CMSCs exhibited an increasing proliferation capacity with the exten-
A
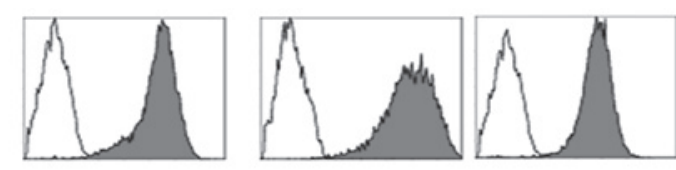

$\mathrm{CD} 73$

CD90

CD105

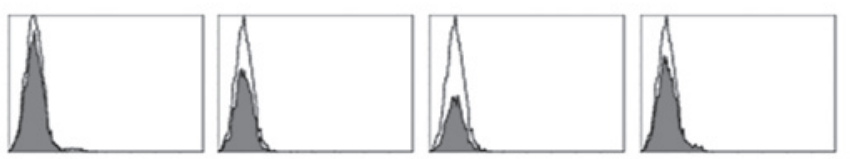

$\mathrm{CD} 14$

$\operatorname{cD} 34$

CD45

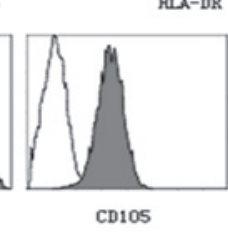

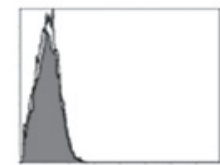

$\mathrm{CD} 14$

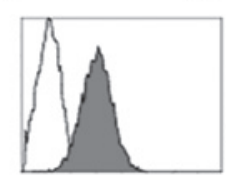

$\mathrm{CD} 73$

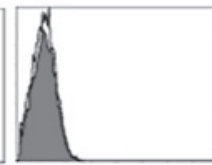

CD34

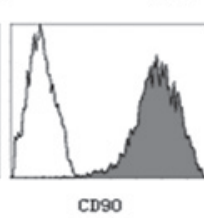

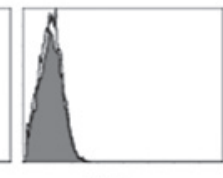

CD45

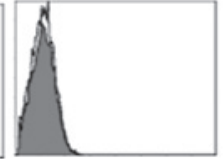

rLA-DR
Figure 4. Flow cytometric analysis of immune phenotypes of (A) amniotic mesenchymal stem cells and (B) chorion mesenchymal stem cells. The white areas indicate the control and the grey areas indicate the treated cells.

sion of culture time. (Fig. 3A) Based on this, the growth curve of the third generation AMSCs and CMSCs was generated, which revealed that the proliferation of AMSCs and CMSCs was not suppressed following passage (Fig. 3B). Therefore, passage three cells (AMSCs and CMSCs) were selected to examine their immune phenotypes. The results of the flow cytometric analysis indicated that the immune phenotypes of AMSCs and CMSCs were consistent with human placental 

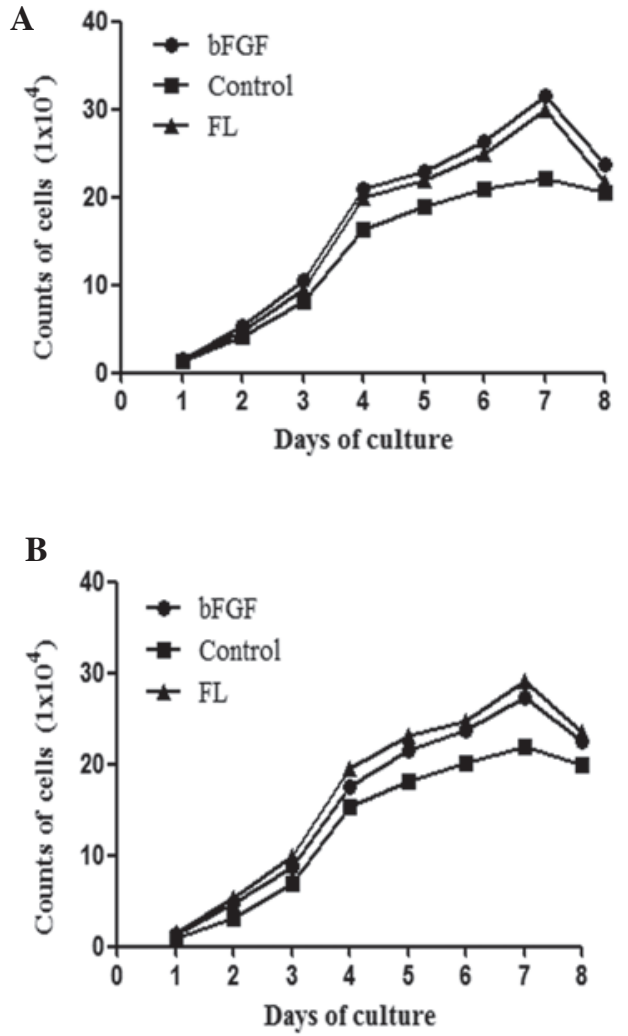

Figure 5. Growth curves of (A) AMSCs and (B) CMSCs cultured with cell factors FL and bFGF. AMSCs, amniotic mesenchymal stem cells; CMSCs, chorion mesenchymal stem cells; FL, fms-related tyrosine kinase 3 ligand.
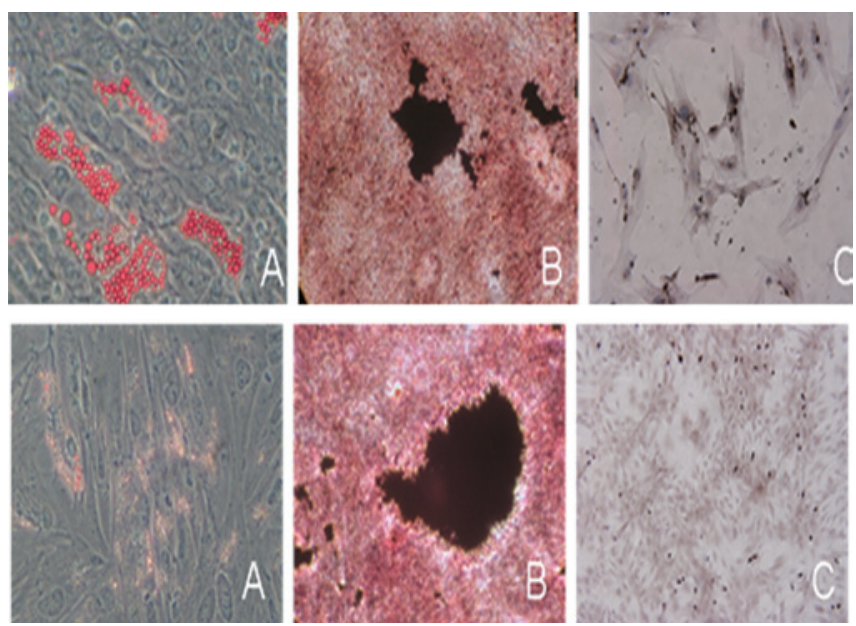

Figure 6. Identification of differentiation of AMSCs and CMSCs into fat, bone and cartilage cells by immunohistochemical analysis following administration of fms-related tyrosine kinase 3 ligand. Differentiation of (top) AMSCs and (bottom) CMSCs into (A) fat cells, oil red O staining, (magnification, x200), (B) bone cells, von Kossa staining (magnification, $\mathrm{x} 100)$ and (C) cartilage, anti-collagen type II immunocytochemistry stain (magnification, x200). AMSCs, amniotic mesenchymal stem cells; CMSCs, chorion mesenchymal stem cells.

MSCs, which were approved for clinical use at an international meeting (10) (Fig. 4).

FL promotes the proliferation of AMSCs and CMSCs. In order to identify the optimal working concentration for each cell factor, different cell factors were tested using an MTT assay.

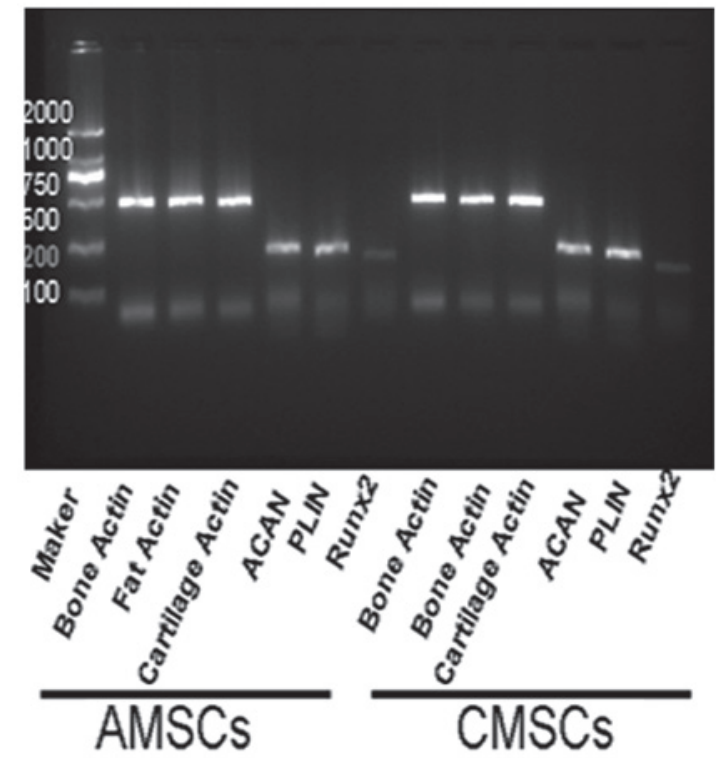

Figure 7. Quantitative polymerase chain reaction analysis of specific gene expression of RUNX2 (osteocytes), PLIN (adipose cells) and ACAN (chondrocytes). RUNX2, runt related transcription factor 2; PLIN, perilipin; ACAN, aggrecan.

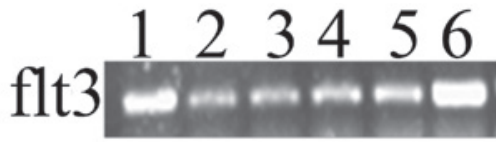

\section{$\beta$-actin ேேேேே・}

Figure 8. Quantitative polymerase chain reaction analysis of Flt3 expression in AMSCs and CMSCs. (1,2, and 3) Flt3 expression in AMSCs which were respectively collected from three puerperas' placenta. (4,5, and 6) Flt3 expression in CMSCs which were respectively collected from three puerperas' placenta. AMSCs, amniotic mesenchymal stem cells; CMSCs, chorion mesenchymal stem cells; flt 3 , fms-related tyrosine kinase 3.

The results demonstrated that all detected cell factors were able to promote the proliferation of CMSCs; however, bFGF and FL demonstrated a higher growth stimulation than others. Thus, the eight-day growth curve of AMSCs and CMSCs was produced (Fig. 5). The results confirmed that FL was able to promote the proliferation of AMSCs and CMSCs; however, the effects were more apparent in CMSCs.

FL affects the immune phenotype of AMSCs and CMSCs. The present study demonstrated that FL was able to promote the proliferation of AMSCs and CMSCs. Furthermore, the present study aimed to identify whether FL was able to alter the immune phenotypes of AMSCs and CMSCs. Therefore, the phenotypes of AMSCs and CMSCs collected from three groups (the control group, bFGF group and FL group) were collected. The results demonstrated that AMSCs and CMSCs highly expressed CD73, CD105 and CD90; however, they did not express CD14, CD34, CD45 and HLA-DR (Tables I and II). This was consistent with the expression profile of human placental MSCs, which were approved for clinical use at an international meeting (10). 
FL impacts the multilineage differentiation potential of AMSCs and CMSCs. In order to gain insight into the multilineage differentiation potential of AMSCs and CMSCs following the administration of FL, the ability of AMSCs and CMSCs to differentiate into fat, bone and cartilage was detected, respectively. The results demonstrated that AMSCs and CMSCs from the FL group were able to differentiate into fat, bone and cartilage. It also indicated that FL not only promoted the proliferation, but also maintained the differentiation potential of MSCs (Fig. 6).

At the same time, qPCR results demonstrated that specific genes, including PLIN (adipose cells), ACAN (chondrocytes) and RUNX2 (osteocytes) were able to be detected following induction of differentiation of AMSCs and CMSCs from the FL group, respectively. The evidence also suggested that FL did not affect the ability of AMSCs and CMSCs to differentiate into fat, cartilage and bone cells (Fig. 7).

Expression levels of Flt3 mRNA in AMSCs and CMSCs. The present study demonstrated that FL was able to promote the proliferation of AMSCs and CMSCs; however, the possible mechanisms remained elusive. It was possibly associated with the expression of its receptor Flt3. Therefore, three different sources of AMSCs and CMSCs were randomly selected from three independent specimens as test objects. The qPCR results demonstrated that Flt3 mRNA was expressed in the AMSCs and CMSCs which were respectively collected from three puerperas' placenta (Fig. 8). This indicated that Flt3 may be important in the process. These experimental data reinforce the important role of FL in promoting the proliferation of AMSCs and CMSCs.

\section{Discussion}

The placental tissue structure and cellular components are relatively complex. Even if non-adherent cells were eliminated through altering the culture solution, the cultured cells may remain mixed with fibroblasts and endothelial cells. Therefore, by shearing placental tissue selectively, it is possible to obtain homogeneous mesenchymal stem cells. In the present study, human placental amnion and chorion were selected for extracting cells. The cells came from the two layers of placenta and were demonstrated to be uniform in our previous experiment and were able to proliferate well in vitro.

The placenta amnion and chorion develop from the neural epiblast progenitors and extra-embryonic mesoderm in embryonic development, respectively. However, due to the different origins of amnion and chorion, and combined with amonion and chorion structural changes in development, it was presumed that the differentiation potentials of amnion and chorion mesenchymal stem cells were different, in addition to their general mesenchymal stem cell characteristics. Further study of the biological characteristics and the molecular mechanisms of mesenchymal stem cells gathering in placental structures, which develop from different germ layers, is important for the use of mesenchymal stem cells in the clinic.

As MSCs become increasingly focused upon, seeking a source which contains MSCs of high quality and at high amounts is clearly important. The present study optimized the culturing conditions on the basis of existing placental mesenchymal stem cell culture technology and selected stem cell factors associated with proliferation, M-CSF, FL and SCF. The present study identified that FL was able to promote the proliferation of AMSCs and CMSCs. FL is the ligand of Flt3 and is mainly expressed in bone marrow stromal cells and certain other cells, which mediates the synthesis of certain growth factors to facilitate the proliferation of stem cells, progenitor cells, dendritic cells and natural killer cells. Flt3 has high sequence homology with the tyrosine kinase receptor family III (RTK III) (13). Flt3, FMS, platelet-derived growth factor receptor (PDGFR) and KIT are all members of the RTK III family and all consist of the five immunoglobulin constituted membrane crossing areas and cytoplasm area, which contains a tyrosine-based motif (14). Flt3 is mainly expressed in infant hemopoietic stem cells, the placenta, gonads and the brain $(15,16)$.

Studies concerning the mechanisms of Flt3 signal transmission preceded FL cloning. Rottapel et al (17) constructed a chimeric molecule of FMS and Flt3 (FF3). When affected by colony stimulating factor 1 receptor (CSF-1), certain Flt3-specific hapten-cell conjugates were detected, whose $\mathrm{C}$ terminal phosphoinositide 3 -kinase has the ability to combine with the $\mathrm{SH} 2$ domain of growth factor receptor-bound protein 2 (17). By combining with CSF-1, FF3 regulates the automatic phosphorylation of tyrosine, leading to a cell mitosis signal. By the Flt3 tissue-specific expression and its signal transmission mechanism, the present study hypothesized that the addition of FL was able to promote the proliferation of placental AMSCs and CMSCs cultured in vitro, and our experimental results supported this hypothesis. In addition, a previous study by our group demonstrated that AMSCs had suitable neurobiological characteristics for being neural precursors and also demonstrated that the transplantation of AMSCs could repair focal cerebral ischemic injury in rats (18). Previous studies by our group concentrated on AMSC-derived microvesicles (MVs), as it had been suggested that tissue regeneration triggered by exogenous stem cells may depend on the release of MVs rather than on stem cell transdifferentiation (19-23).

Through scientific tissue source selection and optimizing MSC culture conditions by the addition of cell factors in order to obtain MSCs at high quality and in large quantities, and examining the biological characteristics of mesenchymal stem cells and their associated molecular regulation mechanisms, the present study may have laid a foundation for further research and clinical application.

\section{Acknowledgements}

This study was supported by the Suzhou Science and Technology Project (nos. SYS201042 and SYS201311).

\section{References}

1. Pittenger MF, Mackay AM, Beck SC, Jaiswal RK, Douglas R, Mosca JD, Moorman MA, Simonetti DW, Craig S and Marshak DR: Multilineage potential of adult human mesenchymal stem cells. Science 284: 143-147, 1999.

2. Rao MS and Mattson MP: Stem cells and aging: expanding the possibilities. Mech Ageing Dev 122: 713-734, 2001.

3. Fernández M, Simon V, Herrera G, Cao C, Del Favero H and Minguell JJ: Detection of stromal cells in peripheral blood progenitor cell collections from breast cancer patients. Bone Marrow Transplant 20: 265-271, 1997. 
4. Erices A, Conget $\mathrm{P}$ and Minguell JJ: Mesenchymal progenitor cells in human umbilical cord blood. Br J Haematol 109: 235-242, 2000.

5. Lee OK, Kuo TK, Chen WM, Lee KD, Hsieh SL and Chen TH: Isolation of multi-potent mesenchymal stem cells from umbilical cord blood. Blood 103: 1669-1675, 2004.

6. Miura M, Gronthos S, Zhao M, Lu B, Fisher LW, Robey PG and Shi S: SHED: stem cells from human exfoliated deciduous teeth. Proc Natl Acad Sci USA 100: 5807-5812, 2003.

7. Romanov YA, Svintsitskaya VA and Smirnov VN: Searching for alternative sources of postnatal human mesenchymal stem cells: candidate MSC-like cells from umbilical cord. Stem Cells 21: 105-110, 2003

8. Yen BL, Huang HI, Chien CC, Jui HY, Ko BS, Yao M, Shun CT, Yen ML, Lee MC and Chen YC: Isolation of multipotent cells from human term placenta. Stem Cells 23: 3-9, 2005.

9. Goodwin HS, Bicknese AR, Chien SN, Bogucki BD, Quinn CO and Wall DA: Multilineage differentiation activity by cells isolated from umbilical cord blood: expression of bone, fat, and neural markers. Biol Blood Marrow Transplant 7: 581-588, 2001.

10. Parolini O, Alviano F, Bagnara GP, et al: Concise review: isolation and characterization of cells from human term placenta: outcome of the first international Workshop on Placenta Derived Stem Cells. Stem Cells 26: 300-311, 2008.

11. Fukuchi Y, Nakajima H, Sugiyama D, Hirose I, Kitamura T and Tsuji K: Human placenta-derived cells have mesenchymal stem/progenitor cell potential. Stem cells 22: 649-658, 2004.

12. Gilliland DG and Griffin JD: The roles of FLT3 in hematopoiesis and leukemia. Blood 100: 1532-1542, 2002.

13. Rosnet $\mathrm{O}$ and Birnbaum D: Hematopoietic receptors of class III receptor-type tyrosine kinases. Crit Rev Oncog 4: 595-613, 1993.

14. Agnès F, Shamoon B, Dina C, Rosnet O, Birnbaum D and Galibert F: Genomic structure of the downstream part of the human FLT3 gene:exon/intron structure conservation among genes encoding receptor tyrosine kinases (RTK) of subclass III. Gene 145: 283-288, 1994.
15. deLapeyrière $\mathrm{O}$, Naquet $\mathrm{P}$, Planche $\mathrm{J}$, Marchetto $\mathrm{S}$, Rottapel $\mathrm{R}$, Gambarelli D, Rosnet O and Birnbaum D: Expression of Flt3 tyrosine kinase receptor gene in mouse hematopoietic and nervous tissues. Differentiation 58: 351-359, 1995.

16. Maroc N, Rottapel R, Rosnet O, Marchetto S, Lavezzi C, Mannoni P, Birnbaum D and Dubreuil P: Biochemical characterization and analysis of the transform ing potential of the FLT3/FLK2 receptor tyrosine kinase. Oncogene 8: 909-918, 1993.

17. Rottapel R, Turck CW, Casteran N, Liu X, Birnbaum D, Pawson T and Dubreuil P: Substrate specificities and identification of a putative binding site for PI3K in the carboxy tail of the murine Flt3 receptor tyrosine kinase. Oncogene 9: 1755-1765, 1994.

18. Li F, Miao ZN, Xu YY, Zheng SY, Qin MD, Gu YZ and Zhang XG: Transplantation of human amniotic mesenchymal stem cells in the treatment of focal cerebral ischemia. Mol Med Report 6: 625-630, 2012.

19. Gatti S, Bruno S, Deregibus MC, Sordi A, Cantaluppi V, Tetta C and Camussi G: Microvesicles derived from human adult mesenchymal stem cells protect against ischemia-reperfusion-induced acute and chronic kidney injury. Nephrol Dial Transplant 26: 1474-1483, 2011.

20. Lai RC, Arslan F, Lee MM, et al: Exosome secreted by MSC reduces myocardial ischemia/reperfusion injury. Stem Cell Res 4: 214-222, 2010.

21. Bruno S, Grange C, Deregibus MC, et al: Mesenchymal stem cell-derived microvesicles protect against acute tubular injury. J Am Soc Nephrol 20: 1053-1067, 2009.

22. Schweitzer KS, Johnstone BH, Garrison J, et al: Adipose stem cell treatment in mice attenuates lung and systemic injury induced by cigarette smoking. Am J Respir Crit Care Med 183: 215-225, 2011

23. Herrera MB, Fonsato V, Gatti S, Deregibus MC, Sordi A Cantarella D, Calogero R, Bussolati B, Tetta C and Camussi G: Human liver stem cell-derived microvesicles accelerate hepatic regeneration in hepatectomized rats. J Cell Mol Med 14: $1605-1618,2010$ 